\title{
Phase-field modeling of the dendrite orientation transition in Al-Zn alloys
}

\author{
Jonathan Friedli, Paolo Di Napoli, Michel Rappaz and Jonathan A. \\ Dantzig
}

Laboratoire de simulation des matériaux, Institut des matériaux, Ecole Polytechnique Fédérale de Lausanne, Station 12 - 1015 Lausanne, Switzerland

E-mail: jonathan.friedli@epfl.ch

\begin{abstract}
With a few exceptions, phase-field simulations of dendritic growth in cubic materials have been modeled using simple expressions for the interfacial energy anisotropy and with strong anisotropy. However, recent experimental results show that the Dendrite Orientation Transition (DOT) observed in Al-Zn alloys by Gonzales and Rappaz [Met. Mat. Trans. A37 (2006) 2797] occurs at weak anisotropy, and modeling these results requires at least two anisotropy parameters. In the present work, we solve the corresponding phase-field model on an adaptive grid, after measuring and compensating for the grid anisotropy. A systematic scan of equiaxed growth simulations was performed in the range of the anisotropy parameter space where the transition is expected. We find separate domains of existence of $\langle 100\rangle$ and $\langle 110\rangle$ dendrites, similar to those previously reported by Haxhimali et al. [Nat. Mat. 5 (2006) $660]$ for pure materials. In the so-called hyperbranched regime, lying between the $\langle 100\rangle$ and $\langle 110\rangle$ regions, we observe a competition between $\langle 100\rangle$ and $\langle 110\rangle$ growth directions, but no seaweed structures. Directional solidification simulations showed the stabilizing effect of the thermal gradient on the twofold splitting of $\langle 110\rangle$ dendrites, and the importance of the choice of anisotropy parameters. We also found a strong dependence between the orientation of the crystal axes with respect to the thermal gradient and the actual growth direction. Finally, 3-dimensional seaweed microstructures were modeled for the first time, demonstrating that this pattern is a result of not only the values of anisotropy parameters, but also a consequence of directional solidification.
\end{abstract}

\section{Introduction}

It is commonly taught that dendrites in cubic metals grow along $\langle 100\rangle$ directions, since these directions correspond to maxima of $\gamma_{s \ell}$. Although this is indeed the case in many systems, such as steel, $\mathrm{Ni}$ - base superalloys, and $\mathrm{Cu}$-base alloys, aluminum alloys display a wider variety of dendrite orientations and morphologies [1-6]. In particular, it has been shown recently that Al-Zn alloys experienced a Dendrite Orientation Transition (DOT) from $\langle 100\rangle$ to $\langle 110\rangle$ when the zinc composition is increased $[7,8]$. This was attributed to a modification of the weak anisotropy of the solid-liquid interfacial energy of aluminum by the strongly anisotropic zinc solute element [9]. In this work, we use phase-field simulations to further explore the role of interfacial energy anisotropy on the selection of dendrite orientation.

We express the interfacial energy $\gamma_{s \ell}$ as an expansion in cubic harmonics [10], given by

$$
\gamma_{s \ell}(\vec{n})=\gamma_{s \ell}^{0}\left[1+a_{1}\left(Q-\frac{3}{5}\right)+a_{2}\left(3 Q+66 S-\frac{17}{7}\right)+\ldots\right]
$$


where $\vec{n}$ is the vector normal to the interface, $\gamma_{s \ell}^{0}$ is the orientation-averaged part of the interfacial energy, and the cubic harmonics $Q$ and $S$ are defined in terms of the Cartesian components of $\vec{n}$ as

$$
Q=n_{x}^{4}+n_{y}^{4}+n_{z}^{4} \quad ; \quad S=n_{x}^{2} n_{y}^{2} n_{z}^{2}
$$

Note that if the series is truncated at the first term, only $\langle 100\rangle$ dendrites can appear. We will show that the observed growth behavior in the Al- Zn system, including the transition between $\langle 100\rangle$ and $\langle 110\rangle$ growth, can be reproduced in simulations when the first two terms in the series are included.

\subsection{Phase-field modeling}

In principle, one could solve the so-called sharp interface problem, in which the interface between the solid and liquid phases evolves through diffusion of heat and solute in the respective phases, and the solid liquid interface satisfies the conditions of local equilibrium of a curved surface. In practice, however, the shape that evolves is too complex to be tracked accurately, especially in 3-D. We use instead the phase-field formalism for binary alloys introduced by Karma [11], and later extended by Echebarria et al. [12]. The model is strictly applicable to the case where the solid diffusivity $D_{s}$ is negligible compared to the liquid diffusivity $D_{\ell}$. We also adopt the "frozen temperature" approximation, in which the temperature field is assumed to be known a priori, which is applicable when the thermal diffusivities in both phases $\alpha_{s, \ell} \gg D_{\ell}$.

The phase-field $\psi \in[-1,1]$ is defined such that $\psi=+1$ corresponds to the solid, $\psi=-1$ to the liquid, and intermediate values are associated with the solid-liquid interface. The concentration $c$ is represented via $\tilde{U}$, the local dimensionless supersaturation with respect to the reference point $c_{\ell}^{0}$, measured in units of the equilibrium concentration gap at that point,

$$
\tilde{U}=\frac{1}{1-k_{0}}\left(\frac{c}{c_{\ell}^{0}}-1\right)
$$

In isothermal solidification, $c_{\ell}^{0}=\left(T_{M}-T_{0}\right) / m_{\ell}$ is the equilibrium concentration of the liquid at a temperature $T_{0}, T_{M}$ is the melting point of the pure substance, and $m_{\ell}$ and $k_{0}$ are the (constant) liquidus slope and partition coefficient, respectively. The evolution equations for $\psi$ and $\tilde{U}$ are given by

$$
\begin{aligned}
& {\left[1-\left(1-k_{0}\right) \tilde{\Theta}\right] \eta^{2}(\vec{n}) \frac{\partial \psi}{\partial \tau}=\nabla\left[\eta^{2}(\vec{n}) \nabla \psi\right] }+\nabla\left(|\nabla \psi|^{2} \eta(\vec{n}) \frac{\partial \eta(\vec{n})}{\partial(\nabla \psi)}\right) \\
&+\psi-\psi^{3}-\lambda\left(1-\psi^{2}\right)^{2}(\tilde{U}+\tilde{\Theta}) \\
&\left(\frac{1+k_{0}}{2}-\frac{1-k_{0}}{2} \psi\right) \frac{\partial \tilde{U}}{\partial \tau}=\nabla\left(\tilde{D}_{\ell} \frac{1-\psi}{2} \nabla \tilde{U}+\vec{j}\right)+\frac{1}{2}\left[1+\left(1-k_{0}\right) \tilde{U}\right] \frac{\partial \psi}{\partial \tau}
\end{aligned}
$$

Eqs. (4) and (5) have been scaled in terms of the nominal interface width $W_{0}$ and relaxation time scale $\tau_{0}$. The scaled diffusivity $\tilde{D}_{\ell}=D_{\ell} \tau_{0} / W_{0}^{2}$. $\tilde{\Theta}=\tilde{x}-\tilde{v}_{P} \tau / \tilde{l}_{T}$ is used to couple the phase-field to the frozen temperature field in directional solidification, where the sample is pulled through a fixed temperature gradient $G$ at velocity $v_{P}$. The dimensionless position along the growth axis $\tilde{x}=x / W_{0}$ is thus reduced by $v_{P} \tau_{0} / W_{0}$ and scaled by the "thermal length" $\tilde{l}_{T}=l_{T} / W_{0}=m_{\ell}\left(k_{0}-1\right) c_{\ell}^{0} / G W_{0} \cdot \eta(\vec{n})$ represents the anisotropic part of the interfacial energy (Eq. 1). Eq. (5) includes an "anti-trapping" current $\vec{j}$, introduced in order to cancel artificial trapping of solute due to the diffuse interface, given by

$$
\vec{j}=\frac{1}{2 \sqrt{2}}\left[1+\left(1-k_{0}\right) \tilde{U}\right] \frac{\partial \psi}{\partial \tau} \frac{\nabla \psi}{|\nabla \psi|}
$$


where $\nabla \psi /|\nabla \psi|$ expresses the normal vector to the interface.

The chemical capillary length $d_{0}=\Gamma_{s \ell} /\left(\left|m_{\ell}\right|\left(1-k_{0}\right) c_{\ell}^{0}\right)$, where $\Gamma_{s \ell}$ is the Gibbs-Thomson coefficient, and the interface attachment coefficient $\beta$ are related to the phase-field parameters via

$$
d_{0}=\alpha_{1} W_{0} / \lambda \quad ; \quad \beta=\alpha_{1} \frac{\tau}{\lambda W_{0}}\left[1-\alpha_{2} \frac{\lambda W_{0}^{2}}{\tau_{0} D}\right]
$$

where $\alpha_{1}=0.8839$ and $\alpha_{2}=0.6267$ are chosen to ensure that the phase-field model converges to the sharp interface model. See Echebarria et al. [12] for further details. Finally, the anisotropy of the interfacial energy is included in the model by defining the interface width $W=W_{0} \eta(\vec{n})$ and the relaxation time $\tau=\tau_{0} \eta^{2}(\vec{n})$. We note that in this model, one cannot guarantee that the kinetic coefficient vanishes, in contrast to the "two-sided" model, where $D_{s}=D_{\ell}$.

The length scales of the grid spacing ( $\Delta x \approx W_{0}$ to resolve the interface) and the simulation domain $\left(L_{x, y, z} \gg D_{\ell} / v\right.$ to avoid interaction between the solute field and the boundaries) are widely different. We therefore solve these equations on an adaptive finite element grid [13-18]. Local error estimators are used to refine or coarsen the mesh where needed, allowing the tracking of the interface as well as the resolution of gradients in the solute fields. Please see the original papers for the details about the adaptive techniques [13]. For the simulations of directional solidification, where initial transients can be very long, we solve the equations in a moving Lagrangian frame that translates at the pulling speed, and the time derivatives in Eqs. (4) and (5) are appropriately augmented with advective terms.

\subsection{Parameter selection}

Unlike the experiments reported in $[7,8]$ where the DOT is observed by changing the $\mathrm{Zn}$ composition, the simulations presented here focus on a single alloy of Al-10 wt pct Zn which presents a relatively short freezing range, hence a rapid development of the structure upon cooling. The anisotropy parameters $a_{1}$ and $a_{2}$ will be varied for this alloy while the other properties are given in Table 1 . The diffusion coefficients were purposely lowered by a factor approximately 2 to allow larger time steps to be used in the explicit solution scheme.

Table 1. Material properties chosen for the phase-field simulations and values found in literature for the composition and temperature considered.

\begin{tabular}{c|c|c|c}
\hline Property & Value in simulation & Literature & Units \\
\hline$c_{0}$ & 0.1 & - & $\mathrm{wt}$ fraction $\mathrm{Zn}$ \\
$m_{\ell}$ & -170 & $-170[19]$ & $\mathrm{K}$ \\
$k_{0}$ & 0.45 & $0.45[19]$ & - \\
$T_{M}$ & 660 & $660[19]$ & ${ }^{\circ} \mathrm{C}$ \\
$D_{\ell}$ & $1.2 \times 10^{-9}$ & $2.75 \times 10^{-9}[20]$ & $\mathrm{m}^{2} / \mathrm{s}$ \\
$D_{s}$ & $1.2 \times 10^{-12}$ & $2.7 \times 10^{-12}[20]$ & $\mathrm{m}^{2} / \mathrm{s}$ \\
$\Gamma_{s \ell}$ & $1.05 \times 10^{-7}$ & $1.05 \times 10^{-7}[21,22]$ & $\mathrm{K} \mathrm{m}$ \\
$\rho L_{f}$ & $1 \times 10^{9}$ & $1.07 \times 10^{9}[22]$ & $\mathrm{J} / \mathrm{m}^{3}$ \\
$\rho c_{p}$ & $3 \times 10^{6}$ & $2.93 \times 10^{6}[23]$ & $\mathrm{J} / \mathrm{m}^{3} \mathrm{~K}$ \\
\hline
\end{tabular}

Echebarria et al. [12] showed that mesh-converged results can be obtained with $W_{0} / d_{0}$ as large as 50 . With the properties above and the assumption that $c_{\ell}^{0}=c_{\infty} / k_{0}=c_{0} / k_{0}$, we have $d_{0} \approx 5 \times 10^{-9} \mathrm{~m}$. The smallest expected tip radius is around $1 \times 10^{-6} \mathrm{~m}$, and using the rule that the tip radius should be at least ten times the interface width, we have $R_{\text {tip }} / W_{0}=10, W_{0}=1 \times 10^{-7} \mathrm{~m}$, and thus $W_{0} / d_{0}=20$. We then obtain $\tilde{D}_{\ell}=5.5$, which implies that $\tau_{0}=4.6 \times 10^{-5} \mathrm{~s}$. All other physical values, such as the thermal length, the pulling 
velocity and the simulation box size are then non-dimensionalized with these values of $W_{0}$ and $\tau_{0}$.

Since our study is focused on the role of anisotropy, it is essential that we measure and correct for grid anisotropy. To do this, we grew dendrites whose crystallographic axes were rotated by various angles with respect to the Cartesian grid. We then measured the difference in growth speed in the different directions, and adjusted the grid anisotropy in order to eliminate the differences. The amount of grid anisotropy depends on the grid spacing. In the calculations given in Section 2, we use $d x_{\min }=0.75 W_{0}$, and grid correction $a_{1}^{\text {grid }}=-0.019$. The time step in the explicit scheme was $0.05 \tau_{0}$.

\section{Results and Discussion}

\subsection{Equiaxed Growth}

Our survey of the anisotropy parameter space for equiaxed growth was performed in one octant of a cubic domain, with all faces having zero-flux boundary conditions. The $\langle 100\rangle$ crystallographic axes were aligned with the Cartesian directions of the grid and an initial one-eighth spherical seed of radius $5-10 \times W_{0}$ was set at one corner. The far-field supersaturation, defined as an input parameter, was either $\Omega=0.4$ or $\Omega=0.25$ in the results presented below. The initial concentration field was then defined as:

$$
c= \begin{cases}k_{o} c_{0} & r<R_{0} \\ c_{0}\left(1+\Omega\left(1-k_{0}\right)\left(\frac{R_{0}}{r}-1\right)\right) & r \geq R_{0}\end{cases}
$$

The computational domain was $768^{3}$ with $d x_{\min }=0.75 W_{0}$. In a uniform grid, this would represent $4.5 \times 10^{8}$ grid points, whereas in our simulations, the grid typically started with about 5000 nodes, and at the end of the simulations, there were $1-4 \times 10^{6}$ nodes. The calculations were run on both 2.93 and $3.30 \mathrm{GHz}$ Intel Xeon CPUs. Run times to generate fully developed microstructures at $\Omega=0.4$ (12000 time steps) were less than one day.

Haxihmali et al. $[9,24]$ examined the DOT in pure materials using phase-field methods. They performed line scans at several fixed values of $a_{2}=(-0.005,-0.01,-0.02)$. At low values of $a_{1},\langle 110\rangle$ dendrites were found, while at high values of $a_{1},\langle 100\rangle$ dominated, and in the intermediate region, mixed structures which they termed "hyperbranched" were obtained. We surveyed the anisotropy parameter space using a similar approach, i.e., using line scans. Since all of the available experimental and MD data [25] show that these parameters are in the range $0 \leq a_{1} \leq 0.12$ and $-0.01 \leq a_{2} \leq 0$, the $\left(a_{1}, a_{2}\right)$ couples were chosen as indicated by Fig. 1 , along with a sampling of the observed structures.

We find similar results to those reported by Haxhimali et al., with respect to the transitions between the three types of microstructures. Looking first at the line scan $a_{2}=-0.01$, we see in Fig. 1 that $\langle 110\rangle$ dendrites form when $a_{1}=0$. Notice that the tip has an elliptic rather than circular shape in a transverse section, reflecting the underlying symmetry of the $\langle 110\rangle$ direction. As $a_{1}$ increases, the strength of the anisotropy along $\langle 100\rangle$ increases, as a result, the $\langle 110\rangle$ tips become more elliptic in (100) planes and are slowed down. If $a_{1}$ is further increased, the tips become sufficiently flat that they split along the $\langle 110\rangle$ axis (see the case $a_{1}=0.04, a_{2}=-0.01$ in Fig. 1). These tips continue to grow, and eventually produce hyperbranched structures. As $a_{1}$ increases along this line scan, the tips split earlier and thus grow larger (cf. $a_{1}=0.06$ ), and then as one approaches the boundary between the hyperbranched and $\langle 100\rangle$ dendrites, the dendrites present four tips split along the $\langle 100\rangle$ axis, until finally they unite into a single tip oriented along the $\langle 100\rangle$.

We note that although the tip growth velocity has converged for the extreme cases with clear $\langle 100\rangle$ and $\langle 110\rangle$ dendrites, the speed of the double and quadruple tips are still in the initial transient at the end of the simulation time considered here. Thus, our simulations should be 


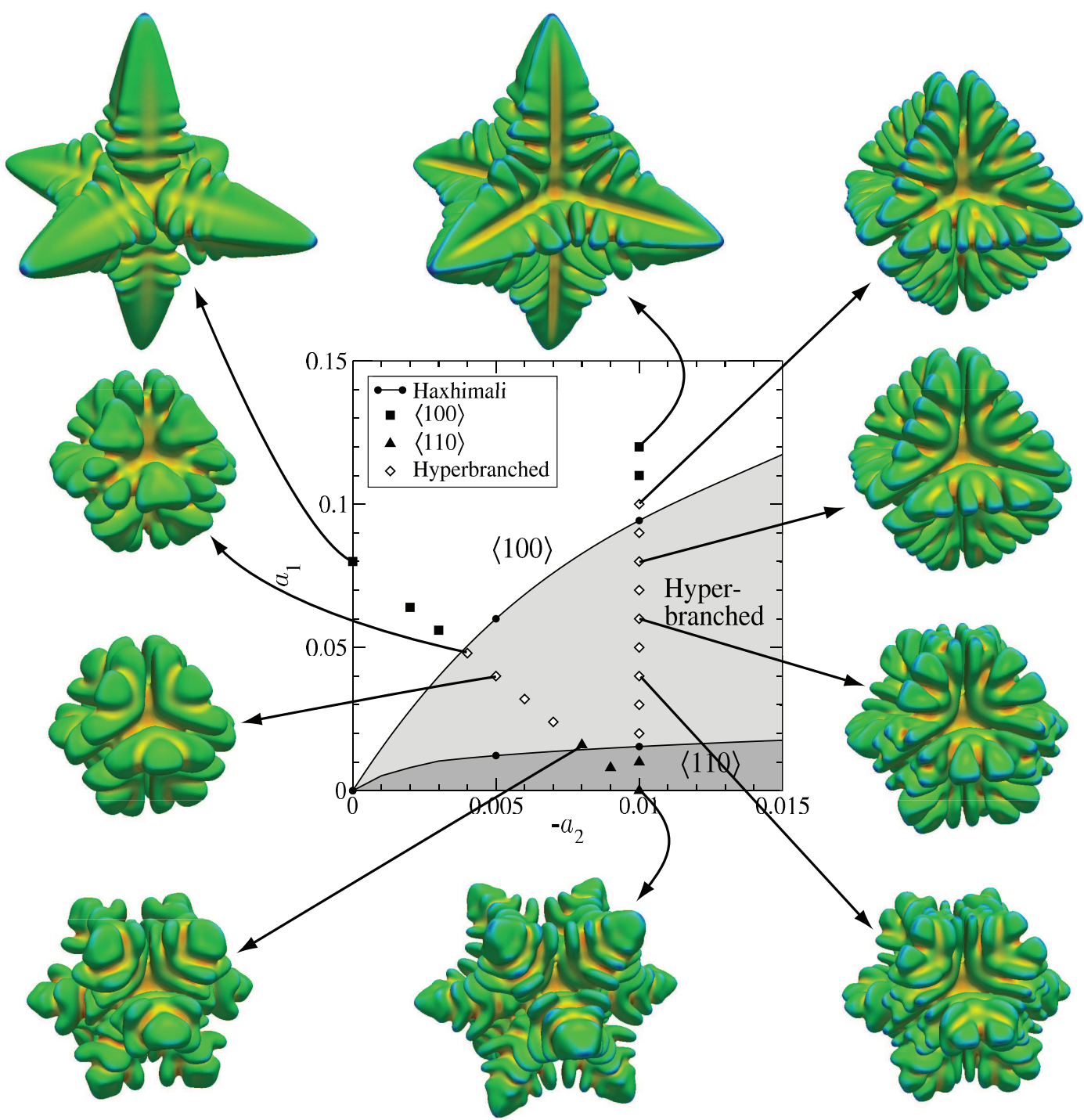

Figure 1. Equiaxed growth phase-field results for varying anisotropy parameters, with $\Omega=0.4$. The scale and orientation of all dendrites is identical. The solid lines in the center graph represent the limits between the domains $\langle 100\rangle$, hyperbranched and $\langle 110\rangle$ structures reported by Haxhimali et al. [9]. The present calculations show very similar results for the locations of the boundaries.

performed for longer times to verify that those structures persist further on and are stable along the found directions at steady state.

Although the locations of the boundaries between the different morphologies are remarkably similar to those reported by Haxhimali et al. for thermal dendrites, the details of the dendritic structures in the hyperbranched region are somewhat different. Haxhimali et al. observed less branching in the off-axis directions. We attribute this difference to the fact that the simulations presented here correspond to solutal dendrites growing at large supersaturation $(\Omega=0.4)$, whereas Haxhimali et al. considered a pure material at very low undercooling, $\Delta=0.05$. Figure 2 compares equiaxed dendrites computed for the parameter set $a_{1}=0.08, a_{2}=-0.01$, using two different supersaturation values, $\Omega=0.4$ and $\Omega=0.25$. Although the envelopes of 
the two dendrites are similar, the amount of "activity" in the $\langle 110\rangle$ directions is significantly less at lower supersaturation. We note that the computations become increasingly expensive as the supersaturation decreases, because the dendrites grow much more slowly, and thus the simulations must be run for a longer time, as noted in the figure caption.

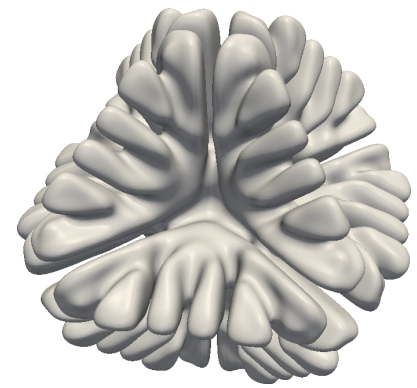

(a) $\Omega=0.4, t=14000$

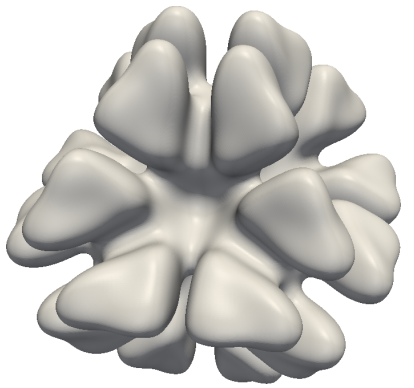

(b) $\Omega=0.25, t=45000$

Figure 2. Comparison of computed equiaxed dendrites with $a_{1}=0.08, a_{2}=-0.01$, at two different supersaturations $\Omega$. The times were chosen from the two simulations such that the dendrite tips were at approximately the same distance from the center.

We would like to be able to present a mechanistic explanation for dendrite orientation selection based on these results. Haxhimali et al. [9] suggested that the dendrites would grow along the direction of minimum interfacial stiffness, which corresponds to the line given by $a_{1}=-20 a_{2} / 3$. Another possible criterion, suggested by Eq. (1), is that there should be transition between $\langle 100\rangle$ and $\langle 110\rangle$ dendrites on opposite sides of the line $a_{1}=-3 a_{2}$, because along this line the coefficient of $Q$ in Eq. (1) is zero. Thus, below this line, the coefficient of $Q$ is negative, which favors $\langle 110\rangle$ dendrites, and above it the coefficient is positive, favoring $\langle 100\rangle$ growth. Both criteria are shown in Fig. 3, superimposed on the tableau of the phase-field results. One can see that although the proposed criteria are suggestive of the selection, clearly they are not predictive.

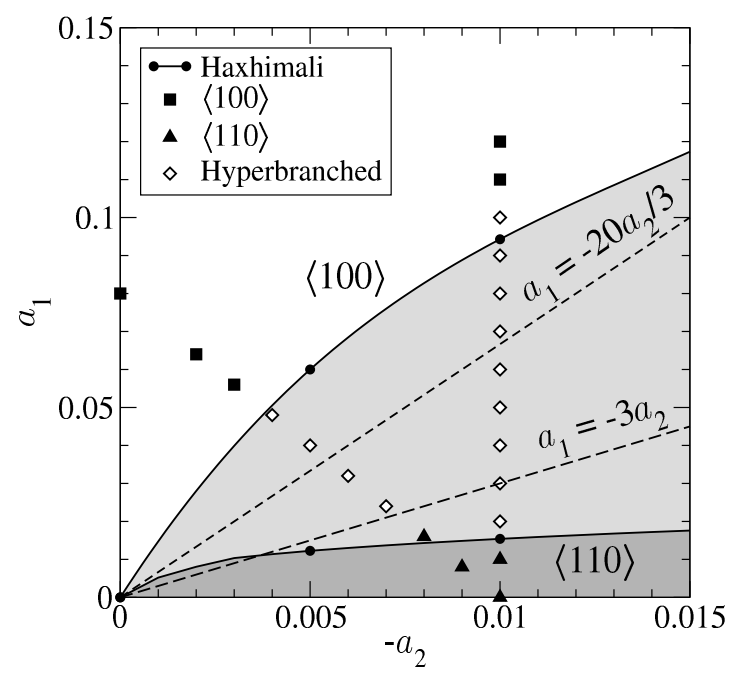

Figure 3. Dendrite growth direction selection criteria based on minimum of the interfacial stiffness (upper dashed line) and maximum of the interfacial energy, Eq. 1 (lower dashed line), superimposed on the results of the phase-field calculations for $\Omega=0.4$, from Fig. 1 . 


\subsection{Columnar Growth}

Directional solidification (DS) simulations were performed in order to obtain a better comparison with the experimental results $[7,26]$, in particular to see if seaweed-type morphologies can be modeled at intermediate zinc compositions (i.e., for anisotropy parameters producing hyperbranched equiaxed dendrites). The parameters used for these simulations are listed in Table 2. These simulations were performed in a rectilinear domain, extended in the pulling direction, which is aligned with the $x$-axis. The $x-z$ faces were periodic, and the $x-y$ faces had symmetry boundary conditions. For cases where we expected to see $\langle 100\rangle$ dendrites, the computational domain in the $y-z$ plane was $192 \times 96 d x_{m i n}$, whereas for cases where we expected to see $\langle 110\rangle$, the computational domain in the $y-z$ plane was $168 \times 48 d x_{\min }$. These dimensions permit a crystal of the corresponding crystalline orientation to grow periodically without being overly constrained by the boundaries.

Table 2. Dimensionless parameters used in the DS simulations, along with their dimensional equivalents. All parameters that are not specifically mentioned here are the same as the values used in the equiaxed growth simulations. $\tilde{v}_{F}$ is the velocity of the reference frame.

\begin{tabular}{c|c|c|c}
\hline $\begin{array}{c}\text { Dimensionless } \\
\text { parameter }\end{array}$ & Value & $\begin{array}{c}\text { Dimensional } \\
\text { equivalent }\end{array}$ & Units \\
\hline$\tilde{l}_{T}$ & $1 \times 10^{4}$ & $G=20777$ & $\mathrm{~K} / \mathrm{m}$ \\
$\tilde{v}_{P}$ & 0.25 & $v_{P}=543$ & $\mu \mathrm{m} / \mathrm{s}$ \\
$\tilde{v}_{F}$ & 0.25 & $v_{F}=543$ & $\mu \mathrm{m} / \mathrm{s}$ \\
$\Omega_{b}$ & 0.45 & - & - \\
\hline
\end{tabular}

In DS, the tip speed is controlled by the pulling velocity. However, as the simulation domain follows the dendrite tip during its growth, we can define a supersaturation $\Omega_{b}$ of the bottom boundary of the domain (or equivalently its temperature). If $\Omega_{b}=1$, and providing the box is long enough along the $x$-direction, the whole mushy zone will be simulated. In order to strike a balance between having enough of the dendrite in the domain to be interesting, and higher computational cost, we chose $\Omega_{b}=0.45$ for the results presented below. We again started with a small spherical seed, this time placed at $(x, y, z)=\left(0, y_{\max } / 2,0\right)$, i.e., along one edge of the base of the domain. Most of the simulations were run in a fixed reference frame $\left(\tilde{v}_{F}=0\right)$ for an initial period to allow the seed to develop, then the simulation was restarted from that point with $\tilde{v}_{F}=\tilde{v}_{P}$.

If the preferred crystallographic growth direction, determined from the simulations of equiaxed growth, was aligned exactly with the pulling direction, the expected dendritic growth patterns appeared. It is more interesting to consider cases where the crystallographic axes are rotated by an angle $\theta$ about the $z$-axis, such that neither the $\langle 100\rangle$ nor the $\langle 110\rangle$ direction is parallel to a coordinate axis. In this way, we can observe the interaction of the preferred growth direction with the constraint imposed by the thermal gradient.

Figures 4 and 5 present results for three cases, with parameter values chosen from values along the diagonal scan in Fig. 1. Each figure shows two fully developed dendrites and two outlined boxes, side-by-side. One of the dendrites is colored by the surface concentration to enhance contrast, whereas the other is simply a reflecting surface. The simulations were performed in just half of one of those boxes, with the results repeated across the periodic face, and reflected across the back face for clarity. Note that this is consistent with the applied periodic and symmetry conditions on the respective faces.

The first case corresponds to the parameter set $a_{1}=0.064, a_{2}=-0.002, \theta=5^{\circ}$. From the results from our study of equiaxed growth, we would expect to find $\langle 100\rangle$ dendrites. Figure 


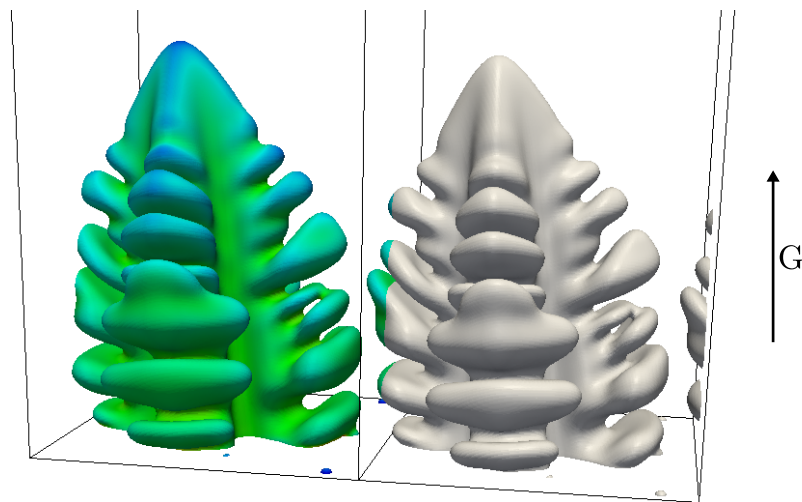

(a) $a_{1}=0.064, a_{2}=-0.002, \theta=5$

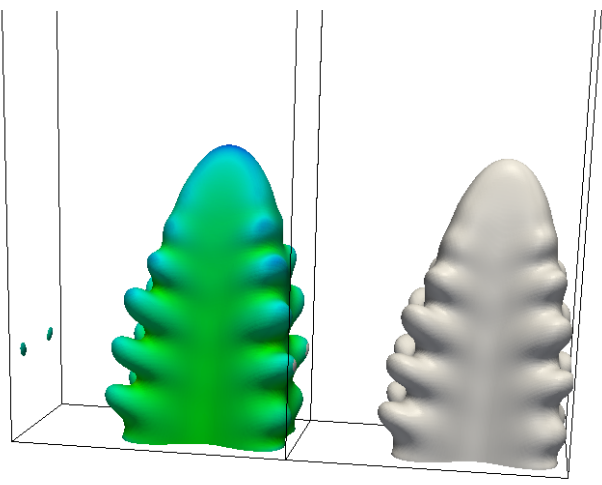

(b) $a_{1}=0.016, a_{2}=-0.008, \theta=40$

Figure 4. Computed DS dendrites for parameter sets that produce (a) $\langle 100\rangle$, or (b) $\langle 110\rangle$ dendrites in equiaxed growth. Note the difference in the size of the computational domain for the two cases.

4(a) shows that the dendrite is indeed of $\langle 100\rangle$ type, but its growth direction is slightly biased towards the direction of the gradient, i.e. between $\langle 100\rangle$ and $\vec{G}$. Note that the combination of the growth non-parallel to the pulling direction and the imposed moving frame causes the dendrite to translate along the $y$-axis, where it re-enters the domain upon reaching the periodic boundary. Figure $4(\mathrm{~b})$ shows the results of simulation with $a_{1}=0.016, a_{2}=-0.008, \theta=40^{\circ}$. This parameter set produced $\langle 110\rangle$ dendrites in equiaxed growth (see Fig. 1). As for the preceding case, this simulation produces the expected orientation, a $\langle 110\rangle$-type dendrite, growing at an intermediate angle between the $\langle 110\rangle$ and the pulling direction, as we saw previously for the $\langle 100\rangle$ dendrite in Fig. 4(a).

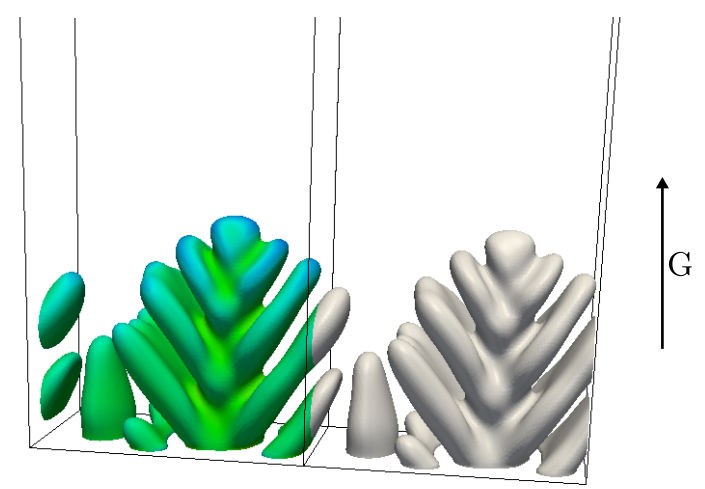

(a) $a_{1}=0.04, a_{2}=-0.005, \theta=40$

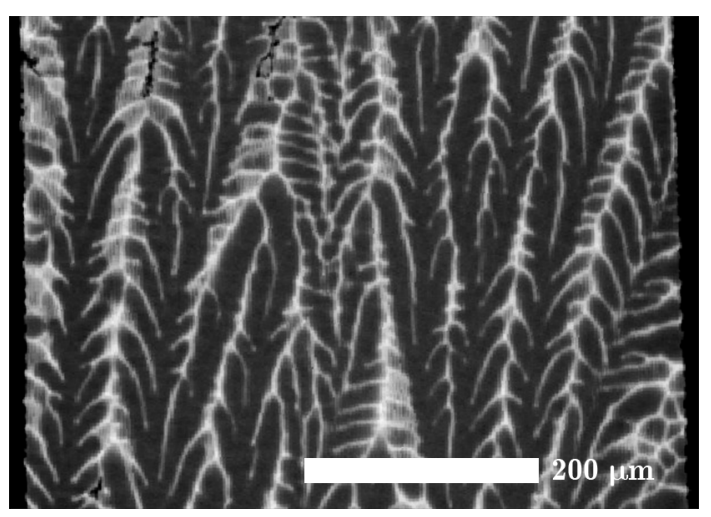

(b) X-ray tomography (100) section

Figure 5. (a) Computed DS dendrites for a parameter set that produces a hyperbranched structure in equiaxed growth. (b) X-ray micro-tomographic section (parallel to a (100) plane) of a directionally solidified Al-50 wt pet Zn alloy.

The most interesting case, shown in Fig. 5(a), corresponds to the parameter set $a_{1}=0.040$, $a_{2}=-0.005, \theta=40^{\circ}$, which lies in the middle of the hyperbranched region in equiaxed growth. The resulting growth pattern produces a primary trunk that tries to follow $\langle 110\rangle$, but continually splits and produces a branch that returns toward the pulling direction. This structure looks remarkably similar to the microstructure obtained from X-ray tomographic measurements on a 
directionally solidified Al-50 wt pct Zn alloy, shown in Fig. 5(b) [26].

\section{Conclusion}

We have presented phase-field calculations for both equiaxed and directional solidification to investigate the role of interfacial energy anisotropy in the selection of dendrite orientation. Working in the range of parameters consistent with experimental measurements, we find distinct regions with $\langle 100\rangle$ and $\langle 110\rangle$ primary dendrites, and an intermediate region where both characters are observed, resulting in hyperbranched dendrites. We show that the hyperbranched structures result from tip splitting of the $\langle 100\rangle$ or $\langle 110\rangle$ primary directions due to the influence of the competing $\langle 110\rangle$ or $\langle 100\rangle$ character, respectively. In directional solidification, the constraints of the imposed thermal gradient and growth direction have a strong effect, especially in the hyperbranched region where neither character is dominant. In particular, in the region of anisotropy parameter space where hyperbranched dendrites are found in equiaxed growth, under DS conditions we observe alternating sidebranches, consistent with experimentally observed microstructures in Al-Zn alloys.

\section{Acknowledgments}

The authors gratefully acknowledge the support of the Swiss National Fund (Grant Nos. 200020113260 and 200020-121598), and the European Community's Seventh Framework Program under grant agreement No. 229108 (MIntWeld), and the US National Aeronautics and Space Administration (NASA) under grant NNX10AJ73G.

\section{References}

[1] Henry S, Jarry P and Rappaz M 1998 Metallurgical and Materials Transactions A: Physical Metallurgy and Materials Science 29 2807-2817

[2] Henry S, Minghetti T and Rappaz M 1998 Acta Materialia 46 6431-6443

[3] Sémoroz A, Durandet Y and Rappaz M 2001 Acta Materialia 49 529-541

[4] Salgado-Ordorica M, Desbiolles J L and Rappaz M 2011 Acta Materialia 59 5074-5084

[5] Salgado-Ordorica M, Burdet P, Cantoni M and Rappaz M 2011 Acta Materialia 59 5085-5091

[6] Niederberger C, Michler J and Jacot A 2006 Physical Review E - Statistical, Nonlinear, and Soft Matter Physics $\mathbf{7 4} 021604$

[7] Gonzales F and Rappaz M 2006 Metallurgical and Materials Transactions A: Physical Metallurgy and Materials Science $\mathbf{3 7} 2797-2806$

[8] Friedli J 2011 Interfacial energy anisotropy and growth morphologies in aluminum-zinc alloys Ph.D. thesis EPFL

[9] Haxhimali T, Karma A, Gonzales F and Rappaz M 2006 Nature Materials 5 660-664

[10] Fehlner W R and Vosko S H 1976 Canadian Journal of Physics 54 2159-2169

[11] Karma A 2001 Physical Review Letters 87115701

[12] Echebarria B, Folch R, Karma A and Plapp M 2004 Physical Review E - Statistical, Nonlinear, and Soft Matter Physics $\mathbf{7 0} 061604$

[13] Jeong J H, Goldenfeld N and Dantzig J 2001 Physical Review E - Statistical, Nonlinear, and Soft Matter Physics 64 416021-4160214

[14] Jeong J H, Dantzig J and Goldenfeld N 2003 Metallurgical and Materials Transactions A: Physical Metallurgy and Materials Science 34 A 459-466

[15] Burman E, Jacot A and Picasso M 2004 Journal of Computational Physics 195 153-174

[16] Provatas N, Greenwood M, Athreya B, Goldenfeld N and Dantzig J 2005 International Journal of Modern Physics B 19 4525-4565

[17] Athreya B, Dantzig J, Liu S and Trivedi R 2006 Philosophical Magazine 86 3739-3756

[18] Narski J and Picasso M 2007 Computer Methods in Applied Mechanics and Engineering 196 3562-3576

[19] Mey S a and Effenberg G 1986 Zeitschrift fuer Metallkunde/Materials Research and Advanced Techniques $77449-453$

[20] Du Y, Chang Y, Huang B, Gong W, Jin Z, Xu H, Yuan Z, Liu Y, He Y and Xie F Y 2003 Materials Science and Engineering A 363 140-151

[21] Morris J, Mendelev M and Srolovitz D 2007 Journal of Non-Crystalline Solids 353 3565-3569 
[22] Valencia J and Quested P 2010 Metals Process Simulation (ASM Handbook vol 22B) (Materials Park, Ohio: ASM International) chap Thermophysical Properties, pp 18-32

[23] Smithells C and Brandes E 1983 Smithells Metals Reference Book (London: Butterworths) chap The physical properties of pure metals sixth ed

[24] Haxhimali T 2006 Phase-field simulation study of dendritic crystal growth morphologies for cubic and hexagonal symmetries Ph.D. thesis Northeastern University

[25] Asta M, Beckermann C, Karma A, Kurz W, Napolitano R, Plapp M, Purdy G, Rappaz M and Trivedi R 2009 Acta Materialia 57 941-971

[26] Friedli J, Fife J, Di Napoli P and Rappaz M 2012 Proceedings of MCWASP 2012 ed Ludwig A 